\section{Fibrous Sepiolite Use As An Asbestos Substitute: Analytical Basics}

\author{
Louis Solebello \\ International Asbestos Testing Laboratories Inc., Mt. Laurel, NJ \\ njlou1960@juno.com
}

\section{Introduction}

Open trade policies have influenced trends in global economic growth. Though difficult to account for all of the effects of this trend, a higher standard of living for previously distressed workers is measurable. This is in part due to acess to previously unavailable technologies. The effects of global economic growth extend further. In many countries, consumers enjoy unparalleled selection of high quality, low cost goods. The environmental impact of global growth is being studied in many ways. One area of study involves analysis and monitoring of product raw materials that many times are not in compliance with environmental regulations of the end user country. Raw material procurement and formulation by manufacturers presents a challenge to the environmental microscopist on several levels. The industrial use of sepiolite and some light microscopy basics of sepiolite is discussed here. Subsequent papers will explore this issue in greater detail as it relates to fibrous mineral analytical choices and techniques available to the environmental microscopist.

\section{Discussion}

In the United States, chrysotile asbestos was previously used in the manufacture of numerous products encountered in every day life. Chrysotile is a naturally occurring fibrous magnesium silicate $\left(\mathrm{Mg}_{3} \mathrm{Si}_{2} \mathrm{O}_{4}(\mathrm{OH})_{4}\right)$ asbestos mineral. It has excellent tensile, slip, and thermal properties often desirable for product beneficiation and performance. Products previously manufactured with Chrysotile include insulations, cements, joint compounds, ceiling tiles, caulks, glazing, brake shoes and gaskets. Current regulations in the United States prohibit the use of Chrysotile and other asbestos minerals due to their carcinonogeneity $^{1,2}$. Manufacturers have been challenged with the task of finding appropriate substitutes as well as monitoring products manufactured overseas for the presence of asbestos.

Sepiolite is a clay mineral $\left(\mathrm{Mg}_{4} \mathrm{Si}_{4} \mathrm{O}_{15} \cdot 6\left(\mathrm{H}_{2} \mathrm{O}\right)\right.$ that can occur in a non-fibrous and fibrous form. The non-fibrous variety has been used to make meerschaum pipes, and is commercially important as a polymer filler as well as a carrier agent for pharmaceutical, catalyst and pesticide applications. The toxicity of sepiolite is not well studied, but current epidemiological research does not indicate that the fibrous form of sepiolite $^{3,4}$ is carcinogenic. As a result, sepiolite is not regulated in the same manner as asbestos and is being more frequently encountered as a chrysotile asbestos substitute in products.

Sepiolite was recently encountered as an asbestos substitute during analysis of gaskets manufactured in China for export to the United States. Some of the gaskets contained sepiolite, whereas others contained chrysotile asbestos and other traditional substitutes such as aramids. The gaskets were not labeled, and MSDS indicating the components used in manufacture of the gaskets were not provided. Examination of the gaskets visually and at low magnifications using a stereomicroscope could not discern which gaskets contained Chrysotile asbestos and which contained sepiolite.

Most environmental laboratories use Polarizing Light Microscopes (PLM) and Transmission Electron

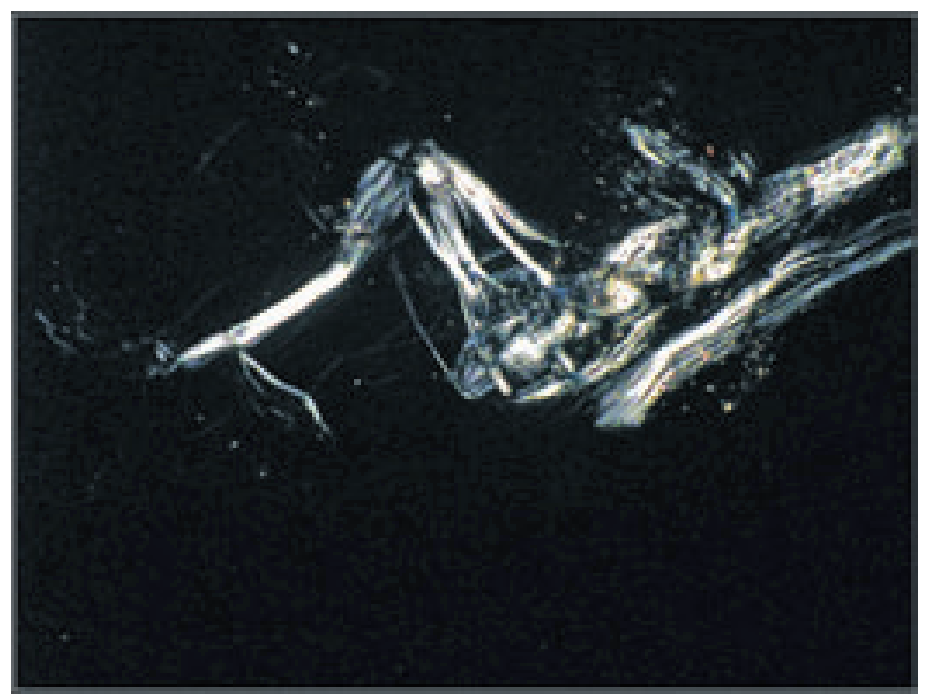

Figure 1. chrysotile asbestos, $100 \times$ magnification, crossed polars. birefringence.

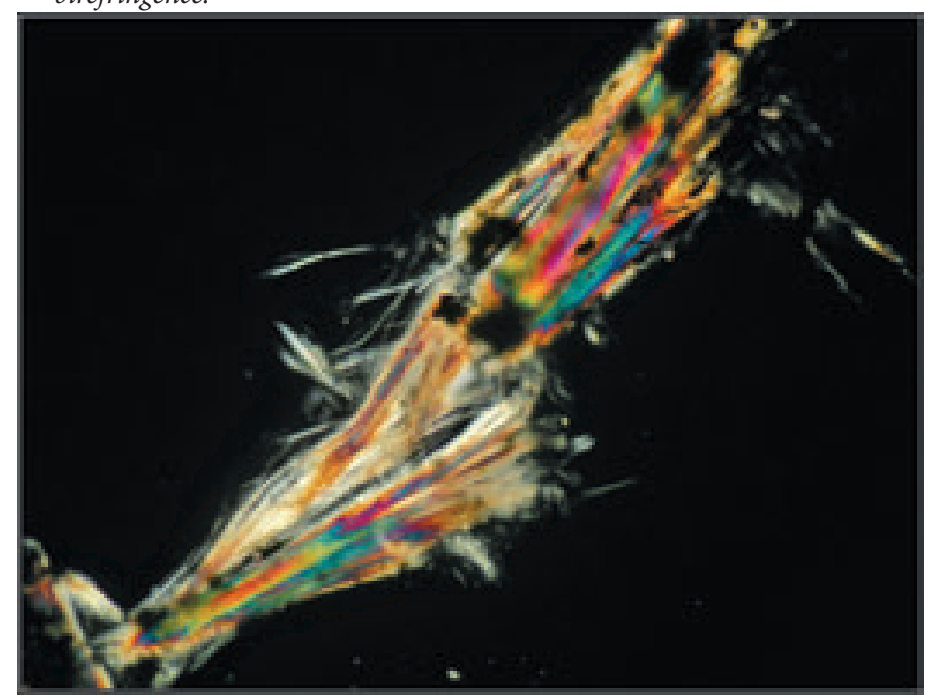

Figure 2. Fibrous sepiolite, 100×, magnification, crossed polars, birefringence.

Microscopes (TEM) for routine analysis of materials for asbestos content. Fibrous sepiolite can present analytical challenges to environmental laboratories during routine (PLM). The asbestiform morphology and birefringence of fibrous sepiolite is similar to chrysotile (figures 1 and 2). Sepiolite refractive indices (RI) are within regulatory accepted ranges reported for chrysotile (Table I). Although slightly lower than most unaltered chrysotile specimens, sepiolite RI's can be mistaken for acid treated chrysotile. As such, analysts unfamiliar with sepiolite might be inclined to identify it erroneously as chrysotile. TEM analysis of sepiolite can present similar misidentifications. Chrysotile and sepiolite have similar compositions, and thus similar Mg:Si EDX ratios (Table I).

\begin{tabular}{|c|c|c|}
\hline Parameter & Chrysotile & Sepiolite \\
\hline Parallel Refractive Index & $1.517-1.567$ & 1.52 \\
\hline Perpendicular refractive Index & $1.493-1.559$ & 1.53 \\
\hline Birefringence & First order $(0.010)^{\mathrm{B}}$ & First Order $(0.001)^{\mathrm{B}}$ \\
\hline Mg: Si Ratio & $7: 10$ & $5: 10$ \\
\hline Cell Dimensions $(\AA)$ & $a=5.313, b=9.12, c=14.64, Z=4$ & $a=13.43, b=26.88, c=5.281$ \\
\hline
\end{tabular}


Figure 3. Sepiolite after application of malachite green stain per Faust procedure. 100× Magnification. Sample in 1.605 refractive index liquid for image contrast using plane polarized light.

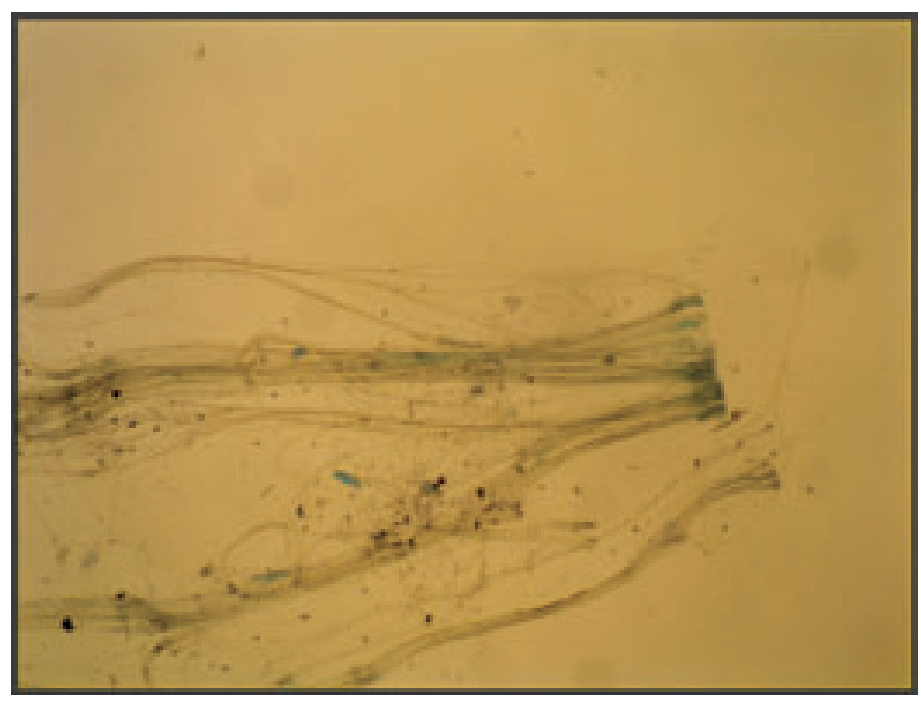

Figure 4. Chrysotile after application of malachite green stain per Faust procedure. 100× Magnification. Sample in 1.605 refractive index liquid for image contrast using plane polarized light

Misidentification can result if a diffraction pattern is not obtained, or if the electron beam does not remain on a fiber long enough for Si peaks to increase relative to $\mathrm{Mg}$ as the mineral fiber degrades. The latter would indicate that the mineral fibers are not chrysotile.

Staining techniques have been used for the identification and differentiation of clays for decades ${ }^{5}$. The most reliable and easiest clay staining techniques to use are acid base aniline dye hydrogen ion indicator tests. Faust ${ }^{6}$ developed an oxidation-reduction technique using malachite green (fast green) in nitrobenzene for differentiating swelling clays (montmorillonites) from non swelling clays (kaolin, illite) that produces artifical pleochorism. This staining technique can be used for rapid differentiation of sepiolite from chrysotile. Suspect fibers are acidified in a drop of $10 \% \mathrm{HCl}$ on a glass microscope slide and heated to evaporation. A drop of $2 \%$ malachite green in nitrobenze is placed on the acidifed mount and rinsed with nitrobenzene until clear. Sepiolite will be stained a green color (Figure 3), whereas chrysotile will not be stained (Figure 4). Preliminary tests using NIST chrysotile treated with $30 \% \mathrm{HCl}$ at ambient temperature for three hours prior did not absorb the malachite green stain using the Faust technique.

\section{Summary}

Constant changes in the global marketplace and mineral fiber regulations will continue to present new challenges to environmental laboratories. New substitutes for old uses of asbestos can present potential misidentifications. The use of sepiolite as a substitute for chrysotile may require additional testing not included within regulatory methods due to the similarity in optical properties and chemistry with chrysotile. Use of acid base hydrogen ion indicator tests such as malachite green by the method of Faust may be useful as an auxiliary technique for differention of sepiolite from chrysotile. Preliminary tests indicate that chrysotile and chrysotile treated with $30 \% \mathrm{HCl}$ for 3 hours do not stain after application of the stain. Further work will detail if chrysotile treated with $30 \% \mathrm{HCL}$ and $\mathrm{H}_{2} \mathrm{SO}_{4}$ at ambient temperature and heated will remain stain resistent. The effects of acid treatments on stain absorption of fibrous sepiolite reference specimens will also be investigated.

\section{References}

1. International Agency for Research on Cancer (March 1998). Asbestos. IARC Monographs on the Evaluation of Carcinogenic Risks to Humans, vol. 14. Lyon, France.

2. U.S. Environmental Protection Agency. Health Effects Assessment for Asbestos. U.S. Environmental Protection Agency, 1984. Environ Health Perspect. 1997 September; 105(Suppl 5): 1049-1052.

3. Investigations on Health-Related Properties of Two Sepiolite Samples. B Bellmann, H Muhle, and H Ernst. Ann. occup. Hyg., Vol. 27, No. 4, pp. 405-414, 1983.

4. In Vitro Tests for the Pathogeneity of Mineral Dusts. Ann. occup. Hyg., Vol. 26, No. 5, pp. 583-592, 1982.

5. Dye Adsorption as a Method of Identifying Clays. Charles C. Dodd,. Clays and Clay Minerals; 1952 v. 1; no. 1; p. 105-111

6. Faust, G.T., Staining of Clay Minerals as a Rapid Means of Identification in Natural and Beneficiated Products. U.S. Bureau of Mines, Report of Investigation of Mines, 3522, 1940.
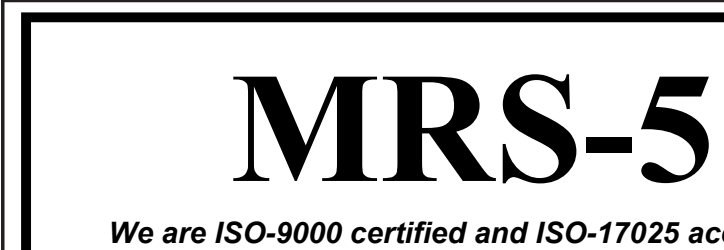

\section{We are ISO-9000 certified and ISO-17025 accredited Microscopy Calibration Standard Now you can calibrate from 1,000X to $1,000,000 X !$}

This is our fourth generation, traceable, magnification reference standard for all types (SEM, FESEM, Optical, STM, AFM, etc.) of microscopy. The MRS-5 has multiple $X$ and $Y$ pitch patterns ranging from $80 \mathrm{~nm}( \pm 1 \mathrm{~nm})$ to $2 \mu \mathrm{m}$ and 3 bar targets from $80 \mathrm{~nm}$ to $3 \mu \mathrm{m}$. There is also a STM test pattern.

Free web resource guide!

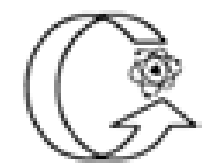

\section{GELLER MICROÅNALYTICAL LABORATORY, Inc.}

426e Boston St., Topsfield, Ma 01983 www.gellermicro.com 